\title{
A presença da fenomenologia na investigação em enfermagem: mapeamento das teses de doutoramento em Portugal
}

Phenomenology in nursing research: mapping of doctoral theses in Portugal

La presencia de la fenomenología en la investigación en enfermería: mapeo de las tesis doctorales en Portugal

Florinda Laura Ferreira Rodrigues Galinha de Sá*(D); Maria Adriana Pereira Henriques** (D);

Maria Antónia Miranda Rebelo Botelho Alfaro Velez ${ }^{* * *}$ (D)

\section{Resumo}

Enquadramento: A fenomenologia progressivamente tem conquistado, no contexto internacional, um espaço de destaque na investigação em enfermagem.

Objetivo: Mapear e caracterizar as teses de doutoramento em enfermagem de abordagem metodológica fenomenológica. Metodologia: Investigação documental, com acesso a 273 teses de doutoramento, realizadas no período de 20042018, disponíveis nos repositórios digitais abertos das universidades portuguesas.

Resultados: Identificaram-se 26 teses de doutoramento com estudos de investigaçáo de abordagem metodológica fenomenológica. A análise realizada teve em conta os seguintes aspetos: a) os participantes - maioritariamente adultos e clientes dos cuidados de enfermagem; b) a área de cuidados de saúde - a maioria das teses náo se enquadra numa área de saúde específica; c) o contexto - primordialmente hospitalar; d) e o método usado - fenomenológico hermenêutico.

Conclusão: As teses de doutoramento, de orientação fenomenológica, embora ainda em percentagem reduzida, ao explicitarem a natureza e o significado da experiência vivida tornam-se relevantes para a construçáo disciplinar de enfermagem.

Palavras-chave: enfermagem; pesquisa qualitativa; hermenêutica; estudos retrospetivos

\section{Abstract}

Background: Phenomenology has been gradually playing a more prominent role in nursing research at an international level.

Objective: To map and characterize nursing doctoral theses with a phenomenological methodological approach.

Methodology: Documentary research, with access to 273 doctoral theses completed between 2004 and 2018 and available in the open-access digital repositories of Portuguese universities.

Results: Twenty-six doctoral theses were identified whose research studies used a phenomenological methodological approach. The analysis took into account the following aspects: a) participants - mainly adults and clients of nursing care; b) healthcare area - most of the theses did not fit into a specific health area; c) context - mainly hospital; d) and research method - phenomenological hermeneutic.

Conclusion: Although still in small numbers, phenomenologically-oriented doctoral theses, by explaining the nature and meaning of lived experiences, become relevant to the development of the nursing discipline.

Keywords: nursing; qualitative research; hermeneutics; retrospective studies

\footnotetext{
*MSc., Professora Adjunta, Escola Superior de Enfermagem de Lisboa, 1600-190, Lisboa, Portugal [fgalinha@esel.pt].(1) https://orcid.org/0000-0002-4523-1721. Contribuiçãono artigo: pesquisa bibliográfica; recolha de dados; tratamento e avaliação estatística; análise de dados e discussão; escrita do artigo. Morada para correspondência: Rua Augusto

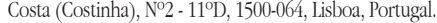

**Ph.D Professora Coordenadora, Escola Superior de Enfermagem de Lisboa, 1600190, Lisboa, Portugal [ahenriques@esel.pt].(B https://orcid.org/0000-0003-0288-6653. Contribuição no artigo: análise de dados e discussão; escrita e revisão do artigo.

**** Ph.D., Professora Coordenadora, Escola Superior de Enfermagem de Lisboa, 1600-

190, Lisboa, Portugal [rbotelho@esel.pt]. (10) https://orcid.org/0000-0001-7356-2053. Contribuição no artigo: análises de dados e discussão; escrita e revisão do artigo.
}

\section{Resumen}

Marco contextual: La fenomenología progresivamente ha conquistado, en el contexto internacional, un espacio de destaque en la investigación en enfermería.

Objetivo: Asignar e caracterizar las tesis de doctorado en enfermería de enfoque metodológico fenomenológico.

Metodología: Investigación documental, con acceso a 273 tesis de doctorado, llevadas a cabo desde 2004 hasta 2018, disponibles en los repositorios digitales abiertos de las universidades portuguesas.

Resultados: Se identificaron 26 tesis de doctorado con estudios de investigación de enfoque metodológico fenomenológico. El análisis realizado tuvo en cuenta los siguientes aspectos: a) los participantes - mayoritariamente adultos y clientes de los cuidados de enfermería; b) el área de cuidado de la salud - la mayoría de las tesis no se ajustan a un área de salud específica; c) el contexto - primordialmente hospitalario; d) y el método usado fenomenológico hermenéutico.

Conclusión: Las tesis doctorales de orientación fenomenológica, aunque todavía en un pequeño porcentaje, al explicar la naturaleza y el significado de la experiencia vivida se vuelven relevantes para la construcción disciplinaria de la enfermería.

Palabras clave: enfermería; investigación cualitativa; hermenéutica; estudios retrospectivos

Recebido para publicação em: 26.06.19

Aceite para publicação em: 18.10 .19

Série IV - n. ${ }^{\circ} 23$ - OUT./NOV./DEZ.2019 


\section{Introdução}

A fenomenologia permite à enfermagem, enquanto ciência, investigar o mundo interior da experiência humana, acedendo aos seus significados pessoais (Chesnay, 2015). A natureza da problemática reforça a necessidade de centrar a prática da investigação em fenómenos humanos relevantes para os cuidados de saúde (Rodriguez, 2018). A fenomenologia permite desocultar a experiência humana circunscrita numa determinada situação e moldada por um contexto único, onde os enfermeiros, enquanto investigadores, são co-construtores de uma realidade social que interessa conhecer para melhor cuidar. A reflexão fenomenológica produz conhecimento sobre o core da experiência humana no âmbito das ciências da saúde, promovendo a humanização dos cuidados de saúde (Rodriguez, 2018).

O doutoramento em enfermagem é uma oportunidade de contribuir para a melhoria da qualidade da prática profissional (Basto, 2016). O doutoramento em enfermagem, enquanto formação científica, pretende dotar os enfermeiros com competências de investigaçáo que os capacitem para produzir conhecimento novo para a área disciplinar de enfermagem. Assim, o avanço ontológico, epistemológico e metodológico da disciplina de enfermagem depende diretamente dos contributos específicos destes investigadores. $\mathrm{O}$ doutoramento em ciências de enfermagem, existe desde 2001, no Instituto de Ciências Biomédicas Abel Salazar (ICBAS) - Universidade do Porto e existe, desde 2004, o programa de doutoramento em enfermagem na Universidade de Lisboa em parceria com a Escola Superior de Enfermagem de Lisboa (ESEL), assim como o curso de doutoramento em enfermagem no Instituto de Ciências da Saúde da Universidade Católica.

A recente entrada da disciplina de enfermagem na academia surge então como um espaço privilegiado de diálogo transdisciplinar entre os saberes das várias disciplinas, sendo promotor do reforço mútuo da investigação científica. A fenomenologia, enquanto metodologia de investigação, tem conquistado progressivamente, no contexto internacional, um espaço de destaque em enfermagem (Chesnay, 2015; Matua, 2015; Moxham \& Patterson, 2017). De tal forma, que num estudo recente de revisão de artigos científicos, publicados em bases de dados europeias, os estudos de metodologia fenomenológica possuem a maior expressão no âmbito da investigação qualitativa em enfermagem (Richards, Hanssen, \& Borglin, 2018). Neste sentido, interessa compreender o estado da arte da investigação em enfermagem, em Portugal, utilizando uma abordagem metodológica fenomenológica. Os objetivos do estudo foram: mapear e caracterizar as teses de doutoramento em enfermagem de abordagem metodológica fenomenológica.

\section{Questão de investigação}

Que produção científica, ao nível do doutoramento de enfermagem, utiliza a abordagem metodológica fenomenológica?

\section{Enquadramento}

O paradigma qualitativo de investigação evoca um prisma naturalista de abordagem da realidade, recorrendo a diversas orientaçôes epistemológicas e metodológicas. A investigação qualitativa em enfermagem procura compreender e interpretar experiências pessoais, interaçóes e contextos sociais para encontrar o sentido da experiência humana (Streubert \& Carpenter, 2013). Por este motivo, a experiência vivida, de um determinado fenómeno no processo de saúde-doença, fornece uma base importante para os cuidados de enfermagem e traz luz para os cuidados de saúde em geral. A fenomenologia emerge num movimento filosófico alemão liderado por Husserl (1859-1938), procurando desvelar a fonte primária da intuição direta do ser em relação aos constituintes essenciais de um fenómeno (Husserl, 2008). A presença da fenomenologia na investigação em enfermagem surge de forma mais emergente no início dos anos 90 (Chesnay, 2015). Historicamente falando é nesta época que surgem investigadores de enfermagem, tais como Diekelmann, Allen e Munhall, que conceptualizam a adaptação da metodologia à disciplina, desafiando os enfermeiros a "pensarem fenomenologicamente" e a terem uma atitude que lhes permita "ser fenomenológicos", mais do que a focarem-se nos aspetos processuais do método fenomenológico (Nelms, 2015). Apesar de atualmente existirem diferentes escolas de fenomenologia, a fenomenologia pode ser essencialmente descritiva, no sentido eidético, ou 
interpretativa, no sentido hermenêutico (Chesnay, 2015). A fenomenologia descritiva, tende, sobretudo, a desocultar a experiência vivida através da utilização de uma descrição pura dos dados da intuição das essências (Beck, 2016). O fenómeno é o que se revela tal como é em si mesmo, como uma essência que se revela numa intuição. Giorgi, reconhecidamente fenomenologista disciplinar, conceptualizou a adaptação do método, proveniente da filosofia, às diferentes disciplinas (Chesnay, 2015). Por outro lado, a fenomenologia interpretativa, tende, sobretudo, a recolocar a centralidade do desocultar da experiência vivida através da interpretação do seu sentido para o Ser (Munhall, 2016). Nesta perspetiva, o investigador não parte da intuição da consciência, mas sim da compreensão da existência do ser no mundo. As duas correntes fenomenológicas, a descritiva e a interpretativa, coexistem ao longo do tempo, sendo que diversos teóricos incrementaram os seus pressupostos filosóficos de base, assim como desenvolveram métodos específicos de análise dos dados $(\mathrm{Mu}-$ nhall, 2016). A adequação entre a problemática em estudo e o percurso metodológico é essencial na fenomenologia (Chesnay, 2015; Rodriguez, 2018). A fenomenologia propóe ao investigador um diálogo interno entre os objetivos da investigação, a perspetiva filosófica adotada e o método de análise de dados, implicando que se percorra um caminho em sintonia desde a explicitação da finalidade do estudo à descrição ou interpretação da experiência vivida. As teses de doutoramento, no âmbito da produção de investigação científica, são documentos de reconhecida qualidade por terem sido alvo de processos de orientação, arbitragem científica e discussão pública. Logo, os estudos de investigação presentes nestes documentos permitem obter um panorama da produçáo científica da disciplina de enfermagem, numa determinada área e período temporal.

\section{Metodologia}

Tendo em conta a questão de investigação e os objetivos do estudo desenhou-se uma pesquisa documental, retrospetiva e descritiva, no período de 2004 a 2018, consultando 273 teses de doutoramento em enfermagem, disponíveis no repositório digital aberto da Universidade de Lisboa (https://repositorio.ul.pt), Universidade Católica Portuguesa (https://repositorio.ucp.pt) e da Universidade do Porto (https://repositorio-aberto. up.pt), no período de janeiro e fevereiro de 2019. Os repositórios digitais institucionais de acesso livre contribuem com uma nova dimensão de divulgação e gestão de informação, permitindo o acesso à produtividade científica (Preto, Martins, Brás, Pimentel, \& Fernández-Sola, 2015). O critério de inclusão foi o estudo, presente nas teses, ser designado, pelo autor, como sendo de abordagem fenomenológica, sendo excluídas as restantes teses. De acordo com os princípios que caracterizam este tipo de investigação, o investigador na análise documental debruça-se sobre o conteúdo presente nos documentos, sendo a análise realizada em consonância com os objetivos da pesquisa (Júnior, Medeiros, \& Augusta, 2017). Procedeu-se à leitura do título e resumo de forma a selecionar as teses de doutoramento que respondiam à questão inicial. A seleção foi validada por todos os autores e as dúvidas decorrentes da análise foram discutidas até ao consenso. Desta forma, como resultado da pesquisa nos repositórios digitais institucionais identificaram-se 26 teses que foram objeto de análise quantitativa descritiva categorial. $\mathrm{Na}$ análise, utilizaram-se as categorias: participantes, área de cuidado de saúde, contexto e método da investigação (Richards et al., 2018), tendo sido também analisadas as palavras-chave das teses e os objetivos dos estudos.

\section{Resultados}

As teses de doutoramento que apresentam estudos de investigação de abordagem metodológica fenomenológica correspondem a $10 \%$ do total das teses, realizadas em Portugal, constituindo ainda uma percentagem muito reduzida. A distribuição anual das teses não foi constante, apresentado variaçóes, sendo o ano de 2011 o que teve a maior frequência com cinco teses (Amorim; Apóstolo; Fabião; Teixeira; Pereira) e em contraste, no ano de 2004 existiu apenas uma (Martins, 2004). Nos anos 2009, 2012, 2013, 2014 e 2017 observa-se de forma consistente a existência de duas teses. De salientar, que $15(57 \%)$ destas teses de doutoramento foram realizadas nos últimos anos, de 2014 a 2018 (Tabela 1). 
Tabela 1

Identificação das teses de doutoramento de abordagem metodológica fenomenológica

\begin{tabular}{|c|c|c|c|}
\hline $\begin{array}{l}\text { Autor/ Ano } \\
\text { Título Tese }\end{array}$ & Objetivo & Palavras-chave & Instituição \\
\hline $\begin{array}{l}\text { Martins, M. M. F. P. S. (2004). O } \\
\text { adulto doente e a familia uma parceria } \\
\text { de cuidados (Tese de Doutoramento). } \\
\text { Recuperado de https://hdl.handle. } \\
\text { net/10216/64647 }\end{array}$ & $\begin{array}{l}\text { Compreender o } \\
\text { fenómeno de assistência } \\
\text { na ótica dos doentes e } \\
\text { seus familiares }\end{array}$ & $\begin{array}{l}\text { Sem } \\
\text { palavras-chave }\end{array}$ & $\begin{array}{l}\text { Universidade do } \\
\text { Porto - Instituto de } \\
\text { Ciências Biomédi- } \\
\text { cas Abel Salazar }\end{array}$ \\
\hline $\begin{array}{l}\text { Ramalho, A. A. (2009). A experiência de } \\
\text { sentir-se respeitada durante o trabalho de } \\
\text { parto no hospital (Tese de Doutoramen- } \\
\text { to). Recuperado de http://hdl.handle. } \\
\text { net/10451/1848 }\end{array}$ & $\begin{array}{l}\text { Compreender a expe- } \\
\text { riência vivida de sentir- } \\
\text {-se respeitada durante o } \\
\text { trabalho de parto }\end{array}$ & $\begin{array}{l}\text { Cuidados de } \\
\text { enfermagem; fe- } \\
\text { nomenologia; hos- } \\
\text { pital; investigação } \\
\text { qualitativa; mãe; } \\
\text { respeito; trabalho } \\
\text { de parto }\end{array}$ & $\begin{array}{l}\text { Universidade de } \\
\text { Lisboa }\end{array}$ \\
\hline $\begin{array}{l}\text { Mendes, I. M. M. M. D. (2009). Ajus- } \\
\text { tamento materno e paterno: Experiências } \\
\text { vivenciadas pelos pais no pós-parto (Tese } \\
\text { de Doutoramento). Recuperado de } \\
\text { https://hdl.handle.net/10216/7250 }\end{array}$ & $\begin{array}{l}\text { Compreender o pro- } \\
\text { cesso de construção do } \\
\text { ajustamento materno e } \\
\text { paterno ao período de } \\
\text { pós-parto, através das } \\
\text { perspetivas das expe- } \\
\text { riências vivenciadas por } \\
\text { ambos os pais }\end{array}$ & $\begin{array}{l}\text { Sem } \\
\text { palavras-chave }\end{array}$ & $\begin{array}{l}\text { Universidade do } \\
\text { Porto - Instituto de } \\
\text { Ciências Biomédi- } \\
\text { cas Abel Salazar }\end{array}$ \\
\hline $\begin{array}{l}\text { Apóstolo, J. L. A. (2011). O imaginário } \\
\text { conduzido no conforto de doentes em con- } \\
\text { texto psiquiátrico (Tese de Doutoramen- } \\
\text { to). Recuperado de https://hdl.handle. } \\
\text { net/10216/7157 }\end{array}$ & $\begin{array}{l}\text { Descrever as vivências } \\
\text { do "conforto-descon- } \\
\text { forto" de doentes inter- } \\
\text { nados em serviços de } \\
\text { psiquiatria clínica }\end{array}$ & $\begin{array}{l}\text { Sem } \\
\text { palavras-chave }\end{array}$ & $\begin{array}{l}\text { Universidade do } \\
\text { Porto - Instituto de } \\
\text { Ciências Biomédi- } \\
\text { cas Abel Salazar }\end{array}$ \\
\hline
\end{tabular}

Reconhecer as representações da mulher sobre a palavra cancro; Conhecer o impacto do Amorim, C. M. B. F. (2011). Doença oncológica da mama: Vivências de mulheres mastectomizadas (Tese de Doutoramento). Recuperado de https://hdl.handle. net/10216/7213

diagnóstico oncológico e da mastectomia na mulher e o seu estado atual após o desenrolar da doença, bem como, conhecer a perceção que a família tem acerca da sua doença

Compreender cada mãe adolescente com base nos significados por ela atribuídos à sua experiência de vida particular, as repercussóes no seu projeto de vida, no seu percurso de vida, nas suas relaçôes familiares e nas interações com os seus pares

$\begin{array}{ll} & \text { Universidade do } \\ \text { Sem } & \text { Porto - Instituto de } \\ \text { palavras-chave } & \text { Ciências Biomédi- } \\ & \text { cas Abel Salazar }\end{array}$

Fabiāo, J. A. S. A. O. (2011). Mães adolescentes: Percursos de vida (Tese de Doutoramento). Recuperado de https:// hdl.handle.net/10216/9172 
Teixeira, M. J. R. (2011). Necessidades de saúde em famílias com jovens adultos (Tese de Doutoramento). Recuperado de https://hdl.handle.net/10216/24593

Pereira, H. J. A. R. (2011). Subitamente cuidadores informais: A experiência de transição para o papel de cuidador informal a partir de um evento inesperado (Tese de Doutoramento). Recuperado de http://hdl.handle.net/10451/3916

Lopes, J. M. O. (2012). Ser cuidado por um enfermeiro gestor de caso: A experiência vivida da pessoa com problemas de adição (Tese de Doutoramento). Recuperado de http://hdl.handle.net/10451/7296

Fernandes, I. M. R. (2012). Quando o enfermeiro se torna doente: Acedendo à experiência vivida (Tese de Doutoramento). Recuperado de http://hdl.handle. net/10451/7423

Coelho, J. C. Q. (2013). Sofrimento e espiritualidade da pessoa com esclerose múltipla (Tese de Doutoramento). Recuperado de http://hdl.handle. net/10400.14/10570
Compreender, junto de estudantes do ensino superior a vivência das suas famílias, para determinar as necessidades em saúde

Compreender o sentido da experiência vivida na transiçáo para o papel de cuidador informal de um convivente significativo a partir de um evento súbito

Compreender a experiência vivida de ser cuidado por um enfer- Pessoa com promeiro gestor de caso, da blemas de adição; pessoa com problemas de adição a substâncias psicoativas cliente da equipa de tratamento do Instituto da Droga e da Toxicodependência

Compreender o significado da experiência vivida de ser doente, na perspetiva individual e única do ser que cuida, na pessoa do enfermeiro

\section{Estudar a vivência do} sofrimento e da espiritualidade das pessoas com esclerose múltipla assim como os meios que estas utilizam para lidar com a doença e sofrimento

Cuidadores informais; experiência vivida; fenomenologia-hermenêutica; Universidade de Lisboa enfermagem
Universidade do

Porto - Instituto de Ciências Biomédicas Abel Salazar tratamento; enfermagem; gestão de caso; experiência vivida

Universidade de Lisboa

Enfermeiros; experiência vivida de Universidade de

doença; fenomeno- Lisboa logia; enfermagem
Universidade Católica Portuguesa - Instituto Ciências da Saúde
Mendes, A. G. P. (2013). Desalojar o Desassossego que vive em si: A necessidade de informação da família na unidade de cuidados intensivos (Tese de Doutoramento). Recuperado de http://bibliografia.bnportugal.gov.pt/bnp/bnp.exe/ registo? 1919063

Nobre, C. F. C. (2014). A experiência da pessoa com dor crónica do foro oncológico: Um estudo fenomenológico (Tese de Doutoramento). Recuperado de http://hdl. handle.net/10400.14/14638

Santiago, M. D. S. (2014). Vivência afetiva em enfermagem (Tese de Doutoramento). Recuperado de http://hdl. handle.net/10400.14/14960
Conhecer na experiência vivida pela família da pessoa adulta na UCI o significado da informação
Família; informação; unidade de cuidados intensivos; experiência vivida; enfermagem
Universidade Católica Portuguesa - Instituto Ciências da Saúde
Compreender como é que a pessoa experiencia a dor crónica do foro oncológico

Esclarecimento conceptual da questão da afetividade no comportamento humano na área da enfermagem
Dor crónica;

doença oncológica; pessoa doente; enfermeiros

Afetividade humana; relaçóes interpessoais; cuidados de enfermagem; ética em enfermagem
Universidade Católica Portuguesa - Instituto Ciências da Saúde

Universidade Católica Portuguesa - Instituto Ciências da Saúde 
Henriques, H. M. S. R. (2015). Experiência do autocuidado e identidade pessoal: Um estudo fenomenológico com pessoas idosas que vivem com doença pulmonar obstrutiva crónica (Tese de Doutoramento). Recuperado de http://hdl. handle.net/10400.14/18573

Ramos, S. E. B. (2015). Perder um irmão até à adolescência: A experiência e o significado na vida adulta (Tese de Doutoramento). Recuperado de http://hdl. handle.net/10400.14/26057
Compreender de que modo a pessoa idosa que vive com DPOC em sua casa é afetada pela experiência de autocuidado na sua identidade pessoal e perspetivar uma assistência de enfermagem holística congruente

Descrever a experiência de perder um irmáo durante a infância e a adolescência, o seu significado para a vida adulta e identificar as implicaçôes para assistência no âmbito da saúde, em particular para a enfermagem. Visando a compreensáo da experiência vivida como foco de atenção para a saúde e para a enfermagem

Rebelo, M. T. S. (2015). O O regresso à vida quotidiana após experiência de uma situação-limite (Tese de Doutoramento). Recuperado de http://hdl.handle. net/10451/18023

Pereira, P. S. (2015). O amor na relação terapêutica em enfermagem: Experiência vivida do enfermeiro de saúde mental (Tese de Doutoramento). Recuperado de http://hdl.handle.net/10451/20503

Lopes, F. M. B. A. (2016). O ambiente hospitalar nos cuidados de enfermagem (Tese de Doutoramento). Recuperado de http://hdl.handle.net/10400.14/20107

\section{Compreender a expe-} riência vivida do regresso à vida quotidiana nos adultos confrontados com uma situação-limite

Compreender a experiência vivida do amor pelos enfermeiros de saúde mental na relação terapêutica em enfermagem

Conhecer a estrutura significativa do conceito ambiente em enfermagem e compreender o fenómeno do ambiente hospitalar nos cuidados de enfermagem

Castro, C. M. C. S. P. (2016). O internamento numa unidade de cuidados intensivos: A experiência vivida da pessoa em situação crítica (Tese de Doutoramento). Recuperado de http://hdl.handle. net/10400.14/21609
Explorar a experiência vivida da pessoa em situação crítica durante o internamento numa UCI
Autocuidado; identidade pessoal; idoso; DPOC; - Instituto Ciências enfermagem
Universidade $\mathrm{Ca}$ tólica Portuguesa da Saúde
Criança;

adolescente;

luto;

luto fraterno;

enfermagem
Situação-limite;

experiência vivida; Universidade de enfermagem; regres- Lisboa so à vida quotidiana
Universidade Católica Portuguesa - Instituto Ciências da Saúde
Amor; relação terapêutica; enfermagem; enfermagem de saúde mental; fenomenologia da prática ambiente; gestâo; pacientes

Universidade Católica Portuguesa - Instituto Ciências da Saúde
Lisboa
Pessoa em situação crítica; unidade de cuidados intensivos; fenomenologia; experiência vivida; enfermagem
Universidade Católica Portuguesa - Instituto Ciências da Saúde

\begin{tabular}{ll}
$\begin{array}{l}\text { Enfermagem; } \\
\text { ambiente; gestão; } \\
\text { pacientes }\end{array}$ & $\begin{array}{l}\text { Universidade Ca- } \\
\text { tólica Portuguesa } \\
\text { - Instituto Ciências } \\
\text { da Saúde }\end{array}$ \\
\hline $\begin{array}{l}\text { Pessoa em situação } \\
\text { crítica; unidade de } \\
\text { cuidados intensivos; } \\
\text { fenomenologia; } \\
\text { experiência vivida; } \\
\text { enfermagem }\end{array}$ & $\begin{array}{l}\text { Universidade Ca- } \\
\text { tólica Pnstituto Ciências } \\
\text { da Saúde }\end{array}$ \\
\hline
\end{tabular}


Rodrigues, S. M. C. (2016). Determinantes e vivências na transição para a parentalidade, em idade reprodutiva tardia, em mães pela primeira vez (Tese de Doutoramento). Recuperado de https:// hdl.handle.net/10216/100586

Magão, M. T. (2017). A esperança em ação: A experiência da esperança em pais de crianças com doença crónica (Tese de Doutoramento). Recuperado de https:// repositorio.ul.pt/handle/10451/40069

Baptista, R. C. N. (2017). Simulação de alta-fidelidade no ensino de enfermagem: Satisfação e ganhos percebidos pelos estudantes (Tese de Doutoramento). Recuperado de https://hdl.handle. net/10216/105288

Marques, M. A. A. (2018). Solitude e sofrimento em pessoas maiores de 80 anos. (Tese de Doutoramento). Recuperado de https://repositorio.ucp.pt/handle/10400.14/27702

Henriques, C. M. G. (2018). Transição para o papel maternal: A experiência vivida de mulheres com problemas de adição a substâncias psicoativas (Tese de Doutoramento). Recuperado de http:// hdl.handle.net/10451/33134

Sousa, M. F. A. (2018). Experiência vivida dos adolescentes com cardiopatia congénita: Uma abordagem fenomenológica (Tese de Doutoramento). Recuperado de http://hdl.handle.net/10451/36930
Compreender as vivências e os determinantes biopsicossociais que envolvem a maternidade em idade reprodutiva tardia em mães pela primeira vez

\section{Compreender a ex-} periência vivida da esperança por pais de crianças com doença crónica.

Analisar as vivências dos estudantes de licenciatura em enfermagem face à participação em experiências clínicas simuladas com simulaçáo de alta-fidelidade (SAF)

Identificar as dimensóes de solidão experienciada pelas pessoas maiores de 80 anos que vivem sós
Gravidez tardia; maternidade tardia; Universidade do transição para a ma- Porto - Instituto de ternidade e parenta- Ciências Biomédilidade; cuidados de cas Abel Salazar enfermagem

Esperança; doença crónica; pais; crian- Universidade de ças; enfermagem; experiência vivida
Lisboa

Simulação de alta-fidelidade; ensino de enfermagem; estudante; satisfação; ganhos
Universidade do Porto - Instituto de Ciências Biomédicas Abel Salazar
Pessoa maior de 80 anos; solitude; sofrimento
Universidade do Porto - Instituto de Ciências Biomédicas Abel Salazar

\section{Compreender a expe-}

riência vivida da transiçấo para o papel maternal, de mulheres com problemas de adição a substâncias psicoativas, desde a gravidez ao primeiro ano de vida do filho.
Transição, maternidade, experiência vivida, adição, fenomenologia, enfermagem
Universidade de

Lisboa

\section{Compreender a expe-} riência vivida dos adolescentes com cardiopatia congénita de acordo com as suas perspetivas e descrever a experiência vivida dos adolescentes com cardiopatia congénita.
Enfermagem; adolescente; cardiopatia Universidade de

congénita; expe- Lisboa riência vivida 
Os participantes dos estudos são maioritariamente adultos (19-64 anos), correspondendo a $61,5 \%$ das teses, contudo sete teses têm participantes adultos e idosos (Amorim, 2011; Castro, 2016; Coelho, 2013; Lopes, 2016; Martins, 2004; Nobre, 2014; Pereira, 2011), duas teses têm participantes exclusivamente idosos (Henriques, 2015; Marques, 2018) e uma tese tem participantes adolescentes (Fabião, 2011). Relativamente ao número de participantes, esta oscila entre três participantes (Rebelo, 2015) e 92 participantes (Amorim, 2011), apresentando as seguintes medidas de tendência central: $\bar{X}=21, M_{o}=10 ; M_{e}=16$. Os participantes dos estudos são sobretudo clientes dos cuidados de enfermagem (85\%), mas também enfermeiros (Fernandes, 2012; Pereira, 2015; Santiago, 2014) e ainda ambos, clientes e enfermeiros (Lopes, 2016).

A área de cuidado de saúde da maioria das teses é referente a outra categoria, pois não se enquadram nas áreas de saúde definidas (Richards et al., 2018), apesar de se terem identificado teses na área de saúde reprodutiva (Fabião, 2011; Henriques, 2018; Mendes, 2009; Ramalho, 2009; Rodrigues, 2016), mental (Apóstolo,
2011; Lopes, 2012; Pereira, 2015), oncológica (Amorim, 2011; Nobre, 2014), neurológica (Coelho, 2013), congénita (Sousa, 2018) e respiratória (Henriques, 2015).

O contexto de cuidados dos estudos predominante é o contexto hospitalar (42\%), incluindo serviços de internamento, consultas externas e hospital de dia. No âmbito da comunidade identificaram-se seis teses (Henriques, 2015; Lopes, 2012; Marques, 2018; Mendes, 2009; Pereira, 2011; Ramos, 2015). De salientar, dentro dos outros contextos, a presença de dois estudos desenvolvidos em escolas superiores de enfermagem (Baptista, 2017; Teixeira, 2011). De forma a ilustrar as temáticas em estudo, intimamente relacionadas com a área de cuidados de saúde e o contexto, destas teses de abordagem metodológica fenomenológica construiu-se uma nuvem de palavras com as suas palavras-chave (Figura 1). A nuvem de palavras mostra a centralidade dos conceitos fenomenologia, enfermagem e experiência-vivida. Também os conceitos doença, cuidados, pessoa e maternidade possuem elevada expressão no universo das palavras-chave das teses de abordagem metodológica fenomenológica.

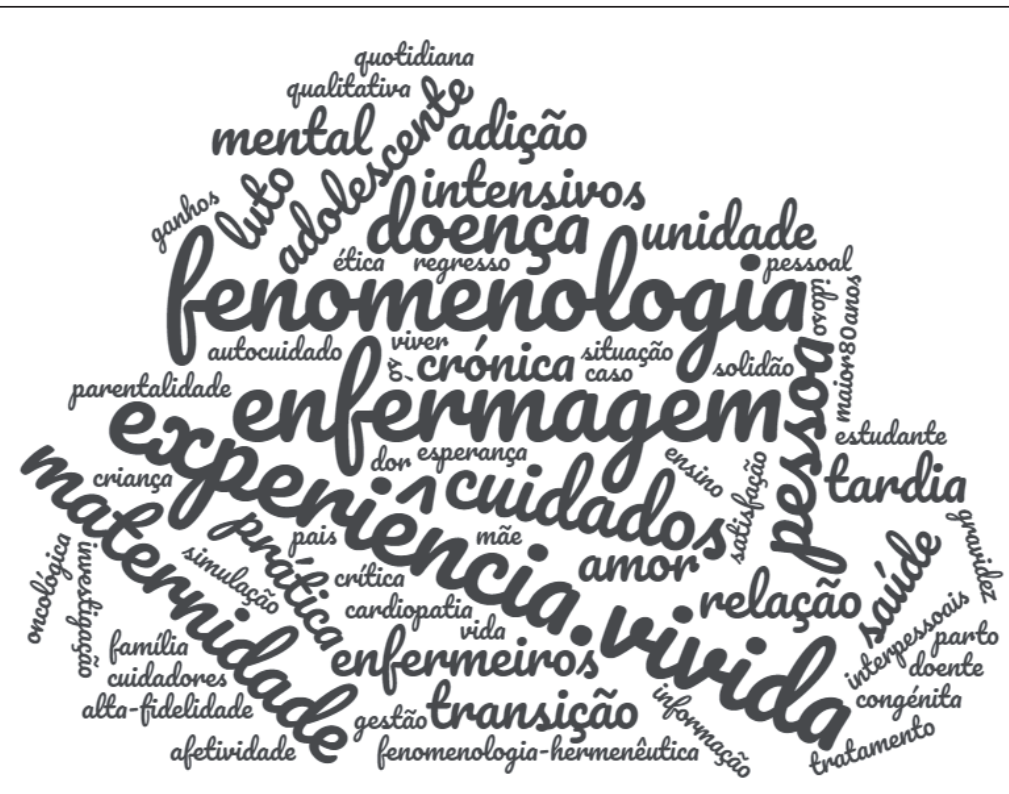

Figura 1. Nuvem de palavras com as palavras-chave das teses. 
Numa análise mais detalhada das teses, tendo por base os objetivos dos estudos, os resultados mostram que mais de metade dos estudos (54\%) define como objetivo o acesso à experiência vivida dos participantes (Castro, 2016; Fabiāo, 2011; Fernandes, 2012; Henriques, 2018; Lopes, 2012; Magão, 2017; Mendes, 2009; Mendes, 2014; Pereira, 2011; Pereira, 2015; Ramalho, 2009; Ramos, 2015; Rebelo, 2015; Sousa, 2018), o que se coaduna com os resultados da nuvem de palavras-chave em que o conceito experiência vivida é central.

As teses de doutoramento analisadas enquadram-se em investigação primária de natureza qualitativa. No total das teses, identificaram-se ainda duas teses com recurso a estudos mistos, em que o estudo qualitativo usava uma abordagem metodológica fenomenológica (Amorim, 2011; Coelho, 2013). Assim como quatro teses baseadas em multiestudos, em que o estudo fenomenológico era habitualmente o primeiro estudo (Apóstolo, 2011; Rodrigues, 2016) ou o segundo estudo (Baptista, 2017; Martins, 2004) de forma a aprofundar a compreensão do fenómeno numa fase inicial da investigação. A principal perspetiva filosófica dos estudos é a da fenomenologia interpretativa ou hermenêutica (Figura 2), com destaque para van Manen (Amorim, 2011; Mendes, 2014; Pereira, 2011; Pereira, 2015; Santiago, 2014; Sousa, 2018). Os estudos com uma abordagem fenomenológica descritiva utilizam sobretudo Giorgi (Baptista, 2017; Castro, 2016; Fernandes, 2012; Mendes, 2009; Ramalho, 2009; Rodrigues, 2016). A categoria outra $(n=7 \%)$ corresponde a teses, usando outras perspetivas fenomenológicas, tais como a fenomenologia existencial e transcendental.

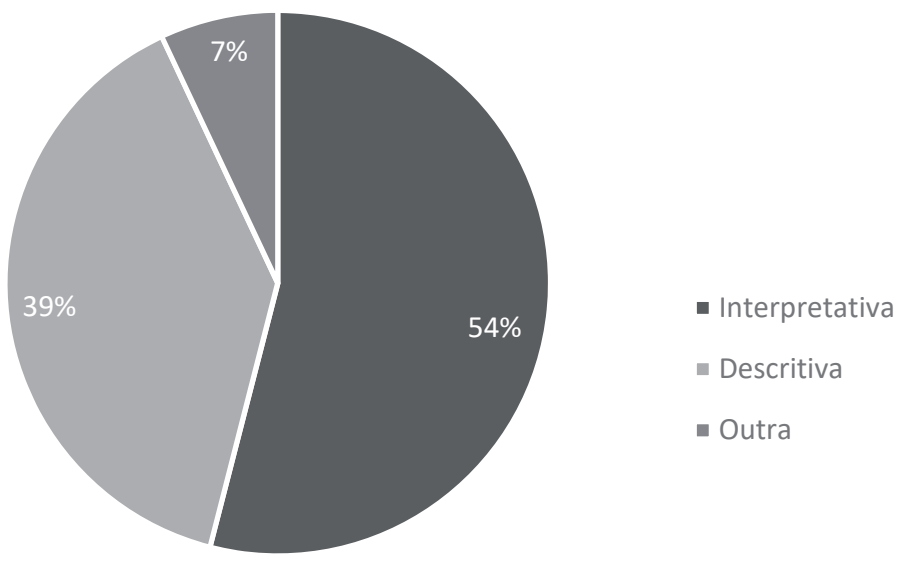

Figura 2. Distribuição dos métodos dos estudos fenomenológicos.

Os estudos fenomenológicos, presentes nas teses, são suportados nas tradiçôes de diferentes perspetivas filosóficas de teóricos de diferentes escolas de fenomenologia.

\section{Discussão}

No universo das teses de doutoramento portuguesas, a presença de estudos de abordagem metodológica fenomenológica é reduzida
$(10 \%)$, sendo que a maioria destas teses de doutoramento foram realizadas nos últimos quatro anos, de 2014 a 2018. A análise realizada refere-se às teses identificadas pelos autores, contudo podem existir outras teses que não estejam disponíveis nos repositórios digitais. Ainda assim, importa referir que num estudo realizado no Brasil, entre 2003 e 2013, consultando teses de enfermagem, identificou-se apenas cerca de $2 \%$ de estudos fenomenológicos (Esquivel et al., 2016). 
Num estudo fenomenológico, comumente o número de participantes é reduzido, porque no processo de seleção dos participantes o investigador procura pessoas com características muito específicas (Chesnay, 2015), ou seja, pessoas que tenham experienciado um determinado fenómeno que interessa conhecer. A dimensão da amostra que mais se observa nestes estudos é a de 10 participantes, dado congruente e expectável face ao habitual reduzido número de participantes nos estudos fenomenológicos. Os participantes são maioritariamente adultos em consonância com os resultados do estudo de revisão europeia de artigos de investigação (Richards et al, 2018). Os achados do presente estudo demonstram também que o principal foco da investigação são os clientes de enfermagem, logo a investigação qualitativa de qualidade emerge como fonte de conhecimento socialmente significativo (Egry \& Fonseca, 2016).

A maioria das teses não se enquadra numa área de saúde específica, pois a maioria dos estudos centram-se em fenómenos associados à vivência do processo de saúde-doença, tais como o luto, a doença crónica, o internamento, a solitude e a transição para o papel de cuidador, não se cingindo a uma área de saúde específica. Contudo, dentro das áreas de saúde identificadas, a área de saúde reprodutiva é a mais emergente, associada a diversas transiçóes que a pessoa e a família vivencia neste processo, o que se coaduna também com os resultados de outros estudos (Esquivel et al., 2016; Richards et al., 2018). O objetivo da fenomenologia é contribuir para uma maior compreensão da natureza e do significado da experiência vivida (van Manen, 2014), pelo que esta fase do ciclo de vida do indivíduo e da família está associada a vivências singulares, como a gravidez, o puerpério e a transição para a parentalidade.

No âmbito das áreas de especialização em enfermagem, presentes em Portugal, existem teses que se enquadram na área de enfermagem médico-cirúrgica (Amorim, 2011; Castro, 2016; Coelho, 2013; Henriques, 2015; Mendes, 2014; Nobre, 2014), na área de enfermagem de saúde materna e obstétrica (Fabião, 2011; Henriques, 2018; Mendes, 2009; Ramalho, 2009; Rodrigues, 2016), na área de enfermagem de saúde mental e psiquiátrica (Apóstolo, 2011; Lopes, 2012; Pereira, 2015), na área de enfermagem de saúde infantil e pediátrica (Magão, 2017;
Ramos, 2015; Sousa, 2018) e na área de enfermagem comunitária (Marques, 2018; Pereira, 2011). A opção por esta reclassificação (Preto, Martins, Brás, Pimentel, \& Fernández-Sola, 2015), permite a análise do predomínio da área de enfermagem médico-cirúrgica, contribuindo os estudos para a compreensão de fenómenos associados à vivência de adultos e idosos com doenças crónicas e incapacitantes, tais como processos de doenças degenerativas e oncológicas. O contexto prevalente é o hospitalar o que também está de acordo com os achados do estudo de revisão europeia de artigos (Richards et al., 2018), contudo destacamos a presença de dois estudos realizados em contexto académico (Baptista, 2017; Teixeira, 2011), sendo os participantes do estudo os estudantes de enfermagem. A realização de investigação fenomenológica em contexto formativo espelha o interesse do corpo docente em compreender melhor a experiência vivida dos estudantes no âmbito dos seus processos pedagógicos.

$\mathrm{O}$ conceito de experiência vivida sendo central num estudo fenomenológico, surge nos objetivos da própria investigação (54\%). Nos restantes $46 \%$, a fenomenologia está presente essencialmente enquanto opção metodológica. Um dos aspetos que torna um estudo fenomenológico, na sua essência, é a articulação profunda na metodologia dos conceitos presentes no fenómeno que se pretende estudar (Norlyk \& Harder, 2010).

A perspetiva fenomenológica interpretativa ou hermenêutica também foi identificada como predominante noutro estudo (Esquivel et al., 2016). Os achados demonstram que dentro desta perspetiva van Manen é o autor mais mobilizado, contudo nesse estudo Heidegger foi o autor predominante dentro da perspetiva hermenêutica (Esquivel et al., 2016). A abordagem da fenomenologia descritiva e da hermenêutica são distintas e requerem do investigador o uso de umas lentes filosóficas específicas no seu caminho de investigação (Chesnay, 2015; Norlyk \& Harder, 2010). A qualidade da investigação qualitativa em enfermagem é necessária no garante do rigor, credibilidade e valor humano da investigação científica (Egry \& Fonseca, 2016). Logo, é crucial na investigação fenomenológica de excelência a clara explicitação da perspetiva filosófica adotada em articulação com o método fenomenológico usado pelos investigadores. 
A presença da abordagem fenomenológica em estudos mistos e multiestudos com abordagem fenomenológica foi surpreendente, correspondendo a cerca de um quarto do total das teses (23\%), o que demonstra a inovação no âmbito da investigação em Portugal, usando a metodologia fenomenológica. Logo, deste estudo emerge uma maior compreensão das opções metodológicas dos investigadores face ao objetivo do estudo, permitindo rastrear um percurso académico na busca da experiência vivida.

\section{Conclusão}

Os estudos fenomenológicos presentes nas teses de doutoramento em enfermagem em Portugal constituem ainda uma percentagem muito reduzida, $10 \%$ dos estudos, contudo a maioria destas teses foi realizada nos últimos anos, de 2014 a 2018. A principal limitação do presente estudo é que ao confinar a pesquisa às teses de doutoramento não permite uma análise abrangente no âmbito da restante produção de investigação científica de enfermagem. Em relação aos objetivos formulados, conclui-se que a maioria das teses tem como objetivo desocultar a experiência vivida de determinado fenómeno através, sobretudo, da fenomenologia interpretativa, recorrendo na sua maioria a participantes adultos e clientes de enfermagem, no contexto hospitalar e na área específica da enfermagem médico-cirúrgica. As teses analisadas dilatam o horizonte de fenómenos humanos como a solitude, a esperança, a situação-limite, o sofrimento, a espiritualidade, o respeito e o amor, pelo que uma maior atenção deve ser direcionada para estes estudos que têm implicaçóes na prática diária dos cuidados. A sedimentação do saber produzido pelos estudos fenomenológicos nas teses de doutoramento em enfermagem, em Portugal, reside numa identidade reflexiva que requer ainda maturidade para ser moldada. A valorização do conhecimento produzido pela fenomenologia centra-se na singularidade da intervenção concreta do cuidado, que se desenvolve e aprofunda naquele contexto específico. Estudos, como o presente, potenciam ainda a discussão entre pares, abrindo os horizontes para os jovens investigadores em enfermagem, para que perspetivem a metodologia fenome- nológica no âmbito da produção científica da licenciatura e mestrado em enfermagem e considerem os novos contextos de ação da enfermagem, dando também visibilidade à experiência vivida do enfermeiro.

\section{Referências bibliográficas}

Basto, M. L. (2016). State of science of qualitative nursing research in Portugal. In C. T. Beck (Ed.), Routledge International Handbook of Qualitative Nursing Research (pp. 514-526). London, UK: Routledge.

Beck, C. T. (2016). Descriptive phenomenology. In C. T. Beck (Ed.), Routledge International Handbook of Qualitative Nursing Research (pp. 133-144). London, UK: Routledge.

Chesnay, M. (2015). Nursing research using phenomenology: Qualitative designs and methods in nursing. New York, NY: Springer.

Egry, E. Y., \& Fonseca, R. M. (2016). Acerca da qualidade nas pesquisas qualitativas em enfermagem. In A. P. Costa (Ed.), Investigação qualitativa: Inovação, dilemas e desafios (4a ed., vol. 2, pp. 75-102). Oliveira de Azeméis, Portugal: Ludomedia.

Esquivel, D., Silva, G., Medeiros, M., Soares, N., Gomes, V., \& Costa, S. (2016). Produção de estudos em enfermagem sob o referencial da fenomenologia. Revista Baiana de Enfermagem, 30(2), 1-10. doi:10.18471/ rbe.v30i2.15004

Husserl, E. (2008). A ideia da fenomenologia (3a ed.). Lisboa, Portugal: Ediçôes 70.

Júnior, E., Medeiros, S., \& Augusta, C. (2017). Análise documental: Uma metodologia de pesquisa para a ciência de informação. Revista Temática, 13(7), 138-150. doiI:10.22478/ufpb.1807-8931.2017v13n7.35383

Matua, G. A. (2015). Choosing phenomenology as a guiding philosophy for nursing research. Nurse Researcher, 22(4), 30-34. doi:10.7748/nr.22.4.30.e1325

Moxham, L., \& Patterson, C. F. (2017). Why phenomenology is increasingly relevant to nurse researchers. Nurse Researcher, 25(3), 6-7. doi:10.7748/nr.25.3.6.s2

Munhall, P. L. (2016). Interpretative phenomenology. In C. T. Beck (Ed.), Routledge International Handbook of Qualitative Nursing Research (pp. 145-161). London, UK: Routledge.

Nelms, T. (2015). Phenomenological philosophy and research. In M. Chesnay (Ed.), Nursing research using phenomenology: Qualitative design and methods in nursing. New York, NY: Springer Publishing Company.

Norlyk, A., \& Harder, I. (2010). What makes a phenomenological study phenomenological? An 
analysis of peer-reviewed empirical nursing studies. Qualitative Health Research, 20(3), 420-431. doi:10.1177/1049732309357435

Preto, L., Martins, M., Brás, M., Pimentel, M., \& Fernández-Sola, C. (2015). Enfermagem portuguesa: Análise da produção e divulgação do conhecimento através de repositórios institucionais. Revista de Enfermagem Referência, 4(6), 35-43. doi:10.12707/RIV14071

Richards, D. A., Hanssen, T. A., \& Borglin, G. (2018). The second triennial systematic literature review of European nursing research: Impact on patient outcome and implications for evidence-based practice.
Worldviews on Evidence-Based Nursing, 15(5), 333343. doi:10.1111/wvn. 12320

Rodriguez, S. J. A. (2018). Phenomenology as a healthcare research method. Evidence-Based Nursing, 21(4), 9698. doi:10.1136/eb-2018-102990

Streubert, H., \& Carpenter, D. (2013). Investigação qualitativa em enfermagem: Avançando o imperativo humanista ( $5^{\mathrm{a}} \mathrm{ed}$.). Loures, Portugal: Lusodidacta.

van Manen, M. (2014). Phenomenology of Practice: Meaning-Giving Methods in Phenomenological Research and Writing. Walnut Creek, EUA: Left Coast Press. 